\title{
Schwer verlaufender Pemphigus foliaceus mit massiver sekundärer Herpes simplex-Superinfektion
}

\author{
Severe Pemphigus Foliaceus with Serious Secondary Herpes Simplex \\ Superinfection
}

Autoren

Institute
R. Renner ${ }^{1}$, M. Sticherling ${ }^{2}$

Klinik für Dermatologie, Venerologie und Allergologie, Universitätsklinikum Leipzig A.ö.R

2 Hautklinik, Universitätsklinikum Erlangen

\section{Bibliografie}

Dol $10.1055 / \mathrm{s}-2008-1077459$

Akt Dermatol 2008; 34:

266-269 @ Georg Thieme

Verlag KG Stuttgart · New York ISSN 0340-2541

Korrespondenzadresse Dr. med. Regina Renner Universitätsklinikum Leipzig Philipp-Rosenthal-Str. 23-25 04103 Leipzig Regina.renner@medizin. uni-leipzig.de

\section{Zusammenfassung \\ $\nabla$}

Wir präsentieren einen Patienten mit prolongiertem und therapierefraktärem Pemphigus foliaceus trotz adäquater immunsuppressiver Medikation. Komplizierend trat eine Herpes simplexSuperinfektion mit beidseitiger Keratitis herpetica sowie Streptokokkenbakteriämie bei bakte-

\section{Anamnese \\ $\nabla$}

Wir berichten im Nachfolgenden über einen damals 60-jährigen Patienten, der im Juli 2002 erstmalig Erytheme mit leichter Schuppung am gesamten Integument bemerkte. Unter der initialen Diagnose einer Tinea corporis erfolgte zunächst die lokale Behandlung mit einer Itrakonazol-haltigen Salbe. Nach fortschreitender Ausbreitung wurden ambulant wiederholt Probeexzisionen durchgeführt und schließlich der Verdacht auf eine Psoriasis vulgaris geäußert. Unter der lokalen Behandlung mit Vitamin D3-Analoga und einer Breitspektrum-UVB-Ganzkörperbestrahlung kam es jedoch zu einer massiven Exazerbation der Hautveränderungen. Weitere Erkrankungen waren bei dem Patienten nicht bekannt, Medikamenteneinnahme wurde verneint.

\section{Erstbefund \\ $\nabla$}

Bei Erstvorstellung in der Klinik befand sich der Patient in leicht reduziertem Allgemeinzustand, aber internistisch unauffälligem und stabilem Status. Am gesamten Integument fielen teils nummuläre, teils großflächig konfluierende hochrote Erytheme und stellenweise schlaffe Blasen mit trüber Flüssigkeit auf ( $\bullet$ Abb. 1), dazu multiple Erosionen und ein periorbitales Ödem. rieller Superinfektion auf. Im weiteren Verlauf erlitt der Patient eine ausgedehnte tiefe Unterschenkelthrombose und Lungenembolie. Diese Kasuistik belegt beispielhaft das hohe Risikopotenzial schwer betroffener Pemphiguspatienten, die durch die immunsuppressive Medikation und konsekutive Erkrankungen zusätzlich zu ihrer Grunderkrankung gefährdet sind.

\section{Befunde diagnostischer Untersuchungen}

$\nabla$

Eine Probebiopsie der Haut zeigte eine superfizielle und perivaskuläre Dermatitis mit Ausbildung subkornealer Kavitäten infolge Akantholyse der Keratinozyten. In der direkten Immunfluoreszenz-Untersuchung zeigten sich interzellulär gebundene IgG-Antikörper mit netzförmigem Muster, die fokal und überwiegend im oberen Anteil der Epidermis lokalisiert waren. Bindung von IgA, IgM oder Ablagerungen von C3 waren nicht nachweisbar. In der indirekten Immunfluoreszenz-Untersuchung auf Affenösophagus fanden sich netzförmige epidermale Ablagerungen von IgG mit einem Titer von 1:160 sowie deutlich erhöhte anti-Desmoglein 1-Ak (92,2 U/ml, NB <20) im ELISA. Desmoglein 3-Ak und andere Autoimmunparameter (Rheumafaktor, anti-SSA-Antikörper) waren unauffällig.

\section{Ergänzende Untersuchungen \\ $\nabla$}

Weiterführende Untersuchungen durch Röntgen des Thorax, Sonografie des Abdomens, Bestimmung von Differenzialblutbild, Serumproteinelektrophorese, Leberenzymen und Nierenretentionsparameter waren unauffällig. 


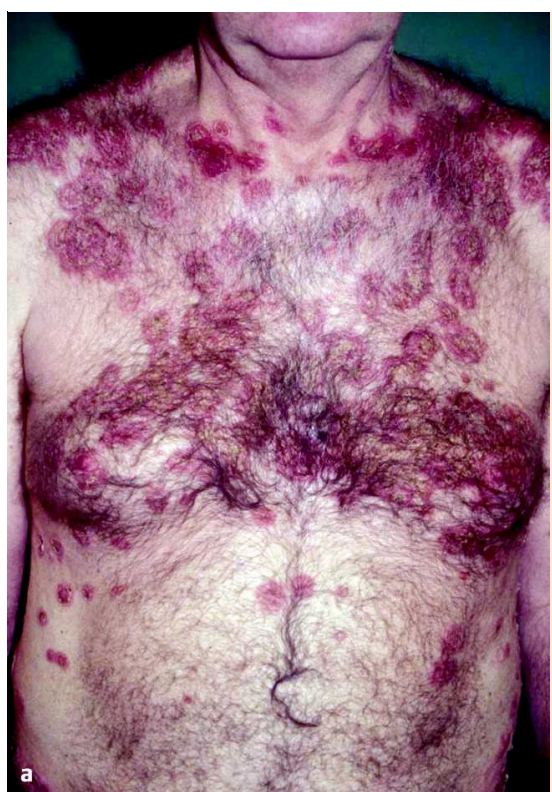

Abb. 1 Befund bei Aufnahme des Patienten: großflächig konfluierende hochrote Erytheme und stellenweise schlaffe Blasen mit trüber Flüssigkeit am Integument, b Detail aus (a).

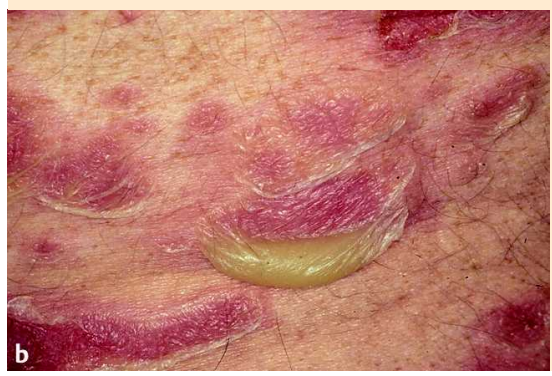

\section{Therapie und Verlauf}

$\nabla$

Aufgrund des klinischen Bildes, der histologischen und immunhistochemischen Veränderungen sowie des serologischen Nachweises von Desmoglein 1-Ak stellten wir die Diagnose eines Pemphigus foliaceus. Initial erfolgte die externe Therapie mit Antiseptika und Glukokortikosteroiden (Klasse II) sowie die Einleitung einer immunsuppressiven Therapie mit Azathioprin $50 \mathrm{mg}$ p.o. (0,5 mg/kgKG) und Prednisolon $1 \mathrm{mg} / \mathrm{kgKG}$ (100 mg) i.v., supportiv mit Osteoporoseprophylaxe und unter Magenschutz. Die Erosionen wurden mit Gaze geschützt und der Patient auf Metalline-Folie gelagert. Dennoch kam es unter dieser Therapie zur Ausbreitung der Erytheme und Zunahme der Blasen, sodass die Dosis von Azathioprin auf $150 \mathrm{mg}(1,5 \mathrm{mg} / \mathrm{kg}$ KG) p.o. und Prednisolon auf $250 \mathrm{mg}$ (2,5 mg/kgKG) i.v. erhöht wurde. Bei weiterhin nur mäßigem Therapieansprechen wurde diese Therapie erweitert um intravenöse Immunglobuline mit einer Gesamtdosis von $2 \mathrm{mg} / \mathrm{kgKG}$ über 1 Woche.

Nach 3 Wochen kam es plötzlich zu einer massiven Verschlechterung des Allgemeinzustandes mit Temperaturen bis $39^{\circ} \mathrm{C}$ bei gleichzeitiger Leukopenie bis 1,7 $\exp ^{9} / 1$ und CRP-Erhöhung auf $233 \mathrm{mg} / \mathrm{dl}$. Betont im Gesichtsbereich, Hals und Dekolleté, aber auch am gesamten Integument zeigten sich multiple bis $2 \mathrm{~cm}$ große konfluierende Ulzerationen und $z$. T. auch schüsselförmige Erosionen ( $\bullet$ Abb. 2). Im Bereich beider Augen zeigte sich eine Konjunktivitis. Ein Zelluloseacetat-Abklatsch bestätigte die Verdachtsdiagnose einer generalisierten Herpes simplex-Infektion mit gleichzeitig vorliegender Keratitis herpetica und beginnenden Vernarbungen. Blutkulturen ergaben eine Bakteriämie mit hämolysierenden Streptokokken der Gruppe B. Daraufhin

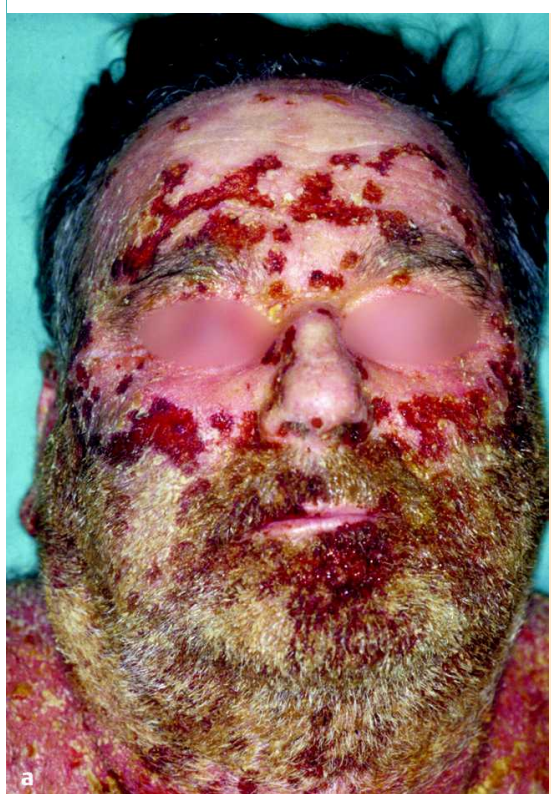

Abb. 2 Dramatische Verschlechterung des Befundes mit zusätzlich bizarr konfluierenden Ulzerationen und z. T. auch schüsselförmigen Erosionen bevorzugt im Kopf und Halsbereich.

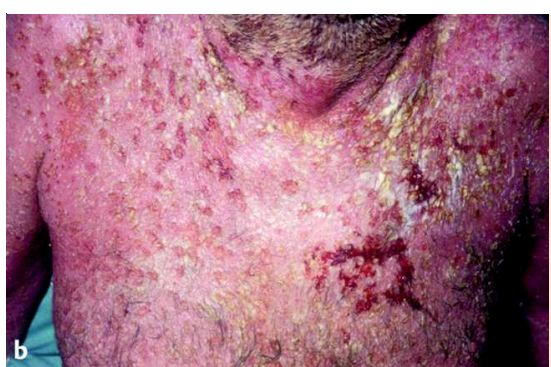

wurde die immunsuppressive Therapie reduziert auf Prednisolon $2 \mathrm{mg} / \mathrm{kgKG}$ (200 mg/Tag) sowie Azathioprin aufgrund der Leukopenie pausiert. Supportiv erhielt der Patient eine Antibiotikatherapie mit Clindamycin $300 \mathrm{mg} 3 \times 1 \mathrm{tgl}$. und Cefuroxim $500 \mathrm{mg} 3 \times 1$ tgl. i.v. gemäß Antibiogramm, eine antivirale Behandlung mit Valtrex $3 \times$ tgl. $500 \mathrm{mg}$ sowie Zovirax-Augensalbe mehrfach täglich. Unter dieser Therapie stabilisierte sich der dermatologische und internistische Befund zügig. Bei wieder normwertigen Leukozyten konnte Azathioprin nach Kontrolle der (normwertigen) Thiopurinmethyltransferase wieder auf $150 \mathrm{mg} /$ Tag gesteigert werden unter gleichzeitiger sukzessiver Reduktion der Prednisolondosis. Die topische Therapie erfolgte weiterhin mit Antiseptika.

9 Tage nach der Entlassung stellte sich der Patient erneut vor mit plötzlich aufgetretener Ruhedyspnoe und Umfangsvermehrung im Bereich beider Beine. Weiterführende Duplex- und CT-Untersuchungen ergaben eine tiefe Beinvenenthrombose rechts mit teilrekanalisierter Thrombose der V. femoralis sowie einer Becken-Beinvenenthrombose der Vena iliaca links. Zusätzlich dazu fand sich eine ausgeprägte beidseitige Lungenembolie mit reitendem Embolus im Bereich der A. pulmonalis, die Emboli ließen sich weit bis in die Segmentarterien verfolgen. Die ausgedehnte Thrombosierung konnte nicht auf eine Thrombophilieneigung zurückgeführt werden, sondern wurde am ehesten als Folge der mehrwöchigen Immobilisierung aufgrund des stationären Aufenthaltes und der hochdosierten Prednisolontherapie angesehen. Es erfolgte eine Antikoagulation mit Phenprocoumon (Falithrom ${ }^{\circledR}$ ) und eine entsprechende Kompressionstherapie. 


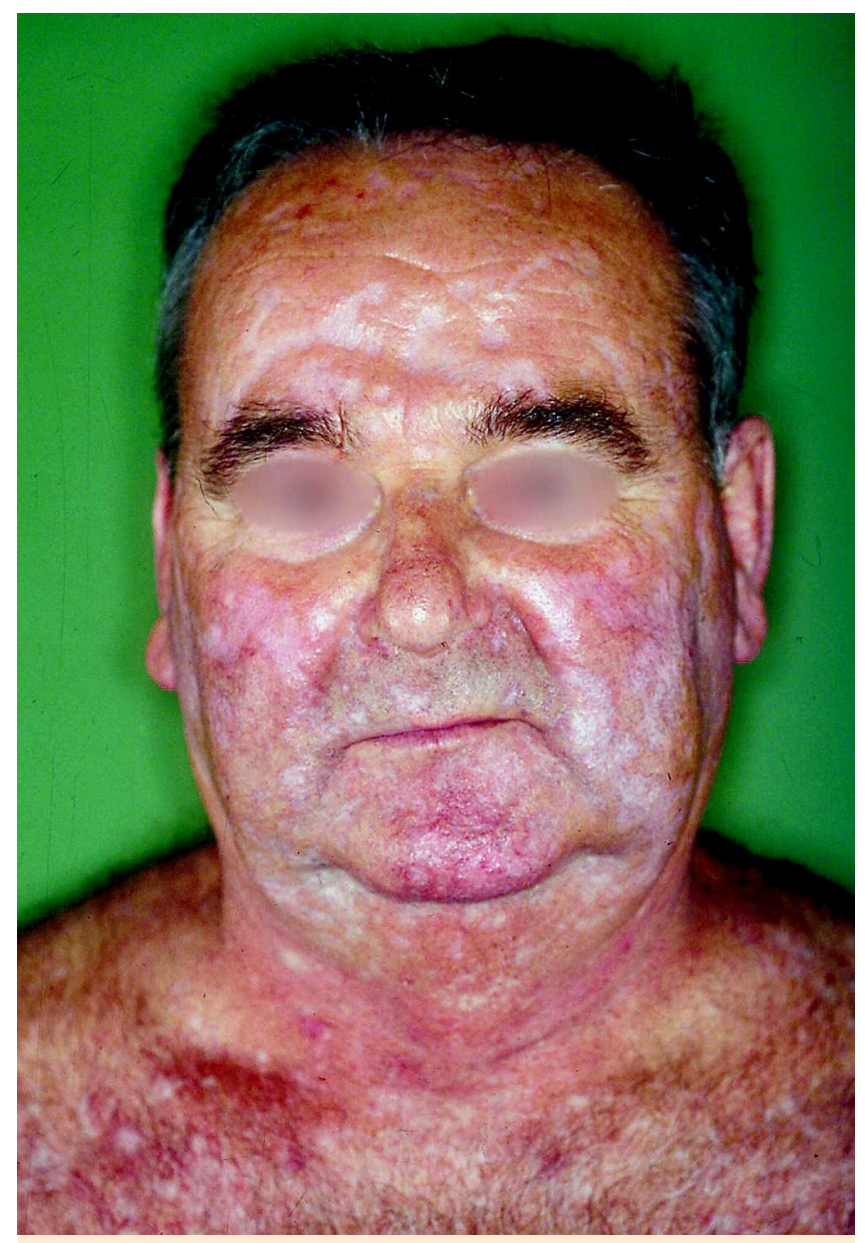

Abb. 3 Befund bei vollständiger Abheilung nach 6 Monaten: Zurück blieben als Folge der superinfizierten Herpesinfektion ausgedehnte hypopigmentierte und atrophe Narben im Gesicht und am Hals.

Im Verlauf nach jetzt 5 Jahren nimmt der Patient derzeit noch $50 \mathrm{mg}$ Azathioprin/Tag ein, ohne dass noch Blasen oder Erosionen auftreten. Da die Autoantikörper gegen Desmoglein 1 mittlerweile auf Normwerte $<20 \mathrm{U} / \mathrm{ml}$ abgesunken sind, soll ein Auslassversuch von Azathioprin erfolgen. Als Folge der superinfizierten Herpesinfektion sind ausgedehnte hypopigmentierte und atrophe Narben im Gesicht und am Hals zurückgeblieben (O Abb. 3).

\section{Diskussion \\ $\nabla$}

Der Pemphigus foliaceus (PF) zählt zu den selteneren Formen der Pemphigusgruppe. Insgesamt wurden seine Prognose und der Krankheitsverlauf verglichen mit dem Pemphigus vulgaris (PV) häufig als milder eingeschätzt. Eine aktuelle Untersuchung zeigte jedoch, dass die Krankheitsaktivität und der Krankheitsverlauf bei PF ebenso schwer ist wie beim PV [1]. Auch in unserem Fall konnten nur hohe und langfristig gegebene Dosen der immunsuppressiven Therapie die Krankheitsaktivität zum Stillstand bringen.

Zum Zeitpunkt der initialen Diagnoseunsicherheit vor stationärer Aufnahme hatte die unter der Verdachtsdiagnose einer Psoriasis vulgaris durchgeführte UV-Therapie zu einer massiven Exazerbation geführt. Ultraviolettes Licht gehört zu den klassischen Triggerfaktoren bei PF und PV und provoziert eine zusätz- liche Akantholyse [2]. Dadurch lässt sich auch die sommerliche Verschlechterung der Erkrankung erklären.

Eine unterschätzte und daher häufig verzögert diagnostizierte Komplikation ist die Superinfektion mit Herpes simplex. Vor allem PF-Patienten scheinen eine Superinfektion zu bekommen, die sich häufig in einer Verschlechterung der Haut oder in scheinbarer Therapieresistenz äußert [3]. Vor allem die primär betroffenen Areale werden vom Herpesvirus befallen, wobei die zunehmende Schmerzhaftigkeit der Hautveränderungen und die gruppiert stehenden Bläschen oder Erosionen auf eine Herpesinfektion hinweisen. Spontanheilungen sind ebenso beschrieben wie letale Fälle $[3,4]$. Gerade bei Patienten mit kombinierter Immunsuppression sollte eine Herpesinfektion bei Verdacht rasch ausgeschlossen oder ggf. behandelt werden. Eine zusätzliche bilaterale Herpeskeratitis wie in unserem Fall ist bisher nur bei einem Patienten mit Pemphigus vulgaris unter Glukokortikoidtherapie beschrieben worden [5].

Systemische Steroide - oft in Kombination mit anderen immunsuppressiven Medikamenten wie Azathioprin, Mycophenolatmofetil, Cyclophosphamid oder Methotrexat gelten als therapeutischer Standard [6]. Sonstige mögliche Optionen sind Rituximab [7] und/oder intravenöse Immunglobuline [8,9]. Vorteil der intravenösen Immunglobulin-Therapie ist ihre gute Verträglichkeit. Diese scheint vor allem bei primären Rituximab-Nonrespondern erfolgreich zu sein [10]. Zur Behandlung therapierefraktärer Fälle wird adjuvant auch die Immunapherese eingesetzt zur Entfernung von Autoantikörpern aus dem peripheren Blut. Außer bei Kontraindikationen sollte zusätzlich eine adjuvante immunsuppressive Therapie erfolgen. Hierunter zeigen die meisten der sonst therapierefraktären Fälle ein gutes bis sehr gutes Ansprechen [11].

\section{Folgerung \\ $\nabla$}

Der Pemphigus foliaceus ist eine seltene Krankheitsvariante der Pemphiguserkrankungen, kann jedoch genauso schwer verlaufen und therapeutische Komplikationen mit sich bringen, die rechtzeitig erkannt und entsprechend behandelt werden müssen. Mögliche physikalische und chemische Triggerfaktoren sind unbedingt zu meiden.

\section{Abstract}

\section{Severe Pemphigus Foliaceus with Serious Secondary Herpes Simplex Superinfection}

We present a patient with prolonged and therapeutically resistant pemphigus foliaceus despite appropriate immunosuppressive treatment. As a severe and life-threatening complication, the patient acquired a herpes simplex superinfection with bilateral keratitis herpetica as well as bacteriemia following streptococcus pyogenes superinfection. After disease control, extensive thrombosis and pulmonary embolism occurred which may be partially caused by the prednisolone therapy. This case demonstrates the high risk potential of patients with pemphigus foliaceus. 


\section{Literatur}

1 Zaraa I, Mokni M, Hsairi M, Boubaker S, Sellami M, Zitouni M, Makni S, Dhahri $A B$. Pemphigus vulgaris and pemphigus foliaceus: similar prognosis? Int J Dermatol 2007; 46: 923-926

2 Reis VM, Toledo RP, Lopez A, Diaz LA, Martins JE. UVB-induced acantholysis in endemi Pemphigus foliaceus (Fogo selvagem) and Pemphigus vulgaris. J Am Acad Dermatol 2000; 42: 571 - 576

3 Zouhair K, el Ouazzani T, Azzouzi S, Sqalli S, Lakhdar H. Herpetic superinfection of pemphigus: 6 cases. Ann Dermatol Venereol 1999; 126: $699-702$

4 Keane JT, Malkinson FD, Bryant J, Levin S. Herpesvirus hominis hepatitis and disseminated intravascular coagulation. Occurrence in an adult with pemphigus vulgaris. Arch Intern Med 1976; 136: 1312 1317

5 Takeshita T. Bilateral herpes simplex virus keratitis in a patient with pemphigus vulgaris. Clin Exp Dermatol 1996; 21: 291 - 292

6 Murrell DF, Dick S, Ahmed AR, Amagai M, Barnadas MA, Borradori L et al. Consensus statement on definitions of disease, end points, and therapeutic response for pemphigus. J Am Acad Dermatol 2008; DOI: 10.1016/j.jaad.2008.01.012

7 Kasperkiewicz M, Zillikens D. Rituximab (anti-CD20) zur Behandlung von bullösen Autoimmundermatosen. Hautarzt 2007; 58: 115-121

8 Segura S, Iranzo P, Martínez-de Pablo I, Mascaró JM, Alsina M, Herrero J, Herrero $C$. High-dose intravenous immunoglobulins for the treatment of autoimmune mucocutaneous blisterung diseases: evaluation of ist use in 19 cases. J Am Acad Dermatol 2007; 56: 960-967

9 Ahmed AR, Sami N. Intravenous immunoglobulin therapy for patients with pemphigus foliaceus unresponsive to conventional therapy. J Am Acad Dermatol 2002; 46: 42 - 49

10 Shimanovich I, Nitschke M, Rose C, Grabbe J, Zillikens D. Treatment of severe pemphigus with protein A immunoabsorption, rituximab and intravenous immunoglobulins. Br J Dermatol 2008; 158: 382 - 388

11 Zillikens D, Derfler K, Eming R, Fierlbeck G, Goebeler M, Hertl M, Hofmann SC, Karlhofer F, Kautz O, Nitschke M, Opitz A, Quist S, Rose C, Schanz S, Schmidt E, Shimanovich I, Michael M, Ziller F. Empfehlungen für die Anwendung der Immunapherese bei der Therapie bullöser Autoimmundermatosen. J Dtsch Dermatol Ges 2007; 5: 881 - 887 